\title{
1 A neuronal prospect theory model in the brain reward circuitry
}

3 Yuri Imaizumi ${ }^{1}$, Agnieszka Tymula $^{2}$, Yasuhiro Tsubo ${ }^{3}$, Masayuki Matsumoto $^{4}$, and Hiroshi

4 Yamada $^{4, *}$

5

$6{ }^{1}$ Medical Sciences, University of Tsukuba, 1-1-1 Tenno-dai, Tsukuba, Ibaraki 305-8577, 7 Japan

$8{ }^{2}$ School of Economics, University of Sydney, Sydney, 2006 NSW, Australia

$9{ }^{3}$ College of Information Science and Engineering, Ritsumeikan University, 1-1-1 Noji-

10 Higashi, Kusatsu, Shiga, 525-8577, Japan

$11{ }^{4}$ Division of Biomedical Science, Faculty of Medicine, University of Tsukuba, 1-1-1 Tenno-

12 dai, Tsukuba, Ibaraki 305-8577, Japan

$14{ }^{*}$ Correspondence to Hiroshi Yamada, Ph.D.

15 Division of Biomedical Science, Faculty of Medicine, University of Tsukuba

16 1-1-1 Tennodai, Tsukuba, Ibaraki, 305-8577 Japan

17 Tel: 81-29-853-6013; e-mail: h-yamada@md.tsukuba.ac.jp

\section{Acknowledgments}

20 The authors would like to thank Takashi Kawai, Ryo Tajiri, Yoshiko Yabana, and Yuki

21 Suwa for their technical assistance, and Jun Kunimatsu and Masafumi Nejime for their

22 valuable comments. Monkey FU was provided by the NBRP "Japanese Monkeys" through

23 the National Bio Resource Project of the MEXT, Japan. Funding: This research was

24 supported by JSPS KAKENHI (Grant Numbers JP:15H05374, 19H05007, and 21H02797), 


\section{Yamada et al.}

25 Takeda Science Foundation, Narishige Neuroscience Research Foundation, Research

26 Foundation for the Electrotechnology of Chubu (H.Y.), JSPS KAKENHI 19K12165 (Y.T.),

27 and ARC DP190100489 (A.T.).

28 Conflict of interest: The authors declare no competing interests.

29 Author Contributions: H.Y. designed the research. H.Y. and Y.I. conducted the

30 experiments. M.M. conducted a part of the experiment. H.Y. and A.T. developed analytic

31 tools. H.Y. and Y.T. conceptualized the simulation tool. H.Y. and A.T. analyzed the data.

32 H.Y., Y.T., and A.T. evaluated the results. H.Y. wrote the first draft. H.Y. and A.T. wrote

33 the manuscript. H.Y., Y.T. wrote a part of the manuscript. All authors edited and approved

34 the final manuscript.

35 Data availability: All data and analysis codes in this study are available in the supporting

36 files (Data_Neuralparameters.csv for the fitted parameters of the best-fit model. Analysis

37 codes: Code_SEUpca.r for the clustering of estimated parameters and Code_Simu.r for

38 the network model simulation). 
Yamada et al.

\section{Summary}

41 Prospect theory, arguably the most prominent theory of choice, is an obvious candidate for

42 neural valuation models. How the activity of individual neurons, a possible computational

43 unit, reflects prospect theory remains unknown. Here, we show with theoretical accuracy

44 equivalent to that of human neuroimaging studies that single-neuron activity in four core

45 reward-related cortical and subcortical regions represents the subjective valuation of risky

46 gambles in monkeys. The activity of individual neurons in monkeys passively viewing a

47 lottery reflects the desirability of probabilistic rewards, parameterized as a multiplicative

48 combination of a utility and probability weighting functions in the prospect theory

49 framework. The diverse patterns of valuation signals were not localized but distributed

50 throughout most parts of the reward circuitry. A network model aggregating these signals

51 reliably reconstructed risk preferences and subjective probability perceptions revealed by

52 the animals' choices. Thus, distributed neural coding explains the computation of

53 subjective valuations under risk.

56 Keywords: prospect theory, reward circuitry, utility, probability weighting, monkey 
Yamada et al.

\section{INTRODUCTION}

59 Prospect theory (Kahneman and Tversky, 1979) proposes that people calculate subjective

60 valuations of risky prospects by a multiplicative combination of their subjective perceptions

61 of two aspects of rewards: a value function that captures the desirability of rewards (i.e.,

62 utility) and an inverse S-shaped probability weighting function (i.e., probability weight) that

63 captures a person's subjective perception of the reward probability. Prospect theory has

64 been the predominant model for describing human choice behavior. The nascent field of

65 neuroeconomics has made significant progress toward an understanding of how the brain

66 makes economic decisions (Camerer et al., 2005; Glimcher et al., 2008); however, many

67 questions remain. One of the fundamental questions is whether discharges from individual

68 neurons follow the prospect theory model.

69 Human neuroimaging provides fundamental insights into how economic decision-

70 making is processed by brain activity, especially in the reward circuitry across cortical and

71 subcortical structures (Haber and Knutson, 2010). This circuitry is thought to learn the

72 values of rewards and the probability of receiving them through experience (Montague et

73 al., 1996; Schultz et al., 1997) and it allows human decision-makers to compute subjective

74 valuations of options. To establish a biologically viable, unified framework explaining

75 economic decision-making, neuroeconomists have applied prospect theory to search for

76 subjective value signals in the human brain using neuroimaging techniques (Hsu et al.,

77 2009; Tobler et al., 2008; Tom et al., 2007). Focusing on the gain domain, previous studies

78 found that the activity of brain regions in the reward circuitry correlates with individual

79 subjective valuations as proposed by the prospect theory (Abler et al., 2006; Berns et al.,

80 2008; Preuschoff et al., 2006; Tobler et al., 2008). However, limitations in temporal and

81 spatial resolutions in neuroimaging techniques have restricted our understanding of how 
Yamada et al.

82 the reward circuitry computes subjective valuations of economic decisions, and there have

83 been almost no studies involving the prospect theory analysis of neural mechanisms in the

84 last decade.

85 Recordings of single-neuron activity in monkeys during gambling behavior may offer

86 substantial progress over existing neuroimaging studies (Abler et al., 2006; Berns et al.,

87 2008; Preuschoff et al., 2006; Tobler et al., 2008). Compared to human research, internal

88 valuation measurements of probabilistic rewards have so far been limited in animals, and

89 not all aspects of the prospect theory model could have been measured (e.g., (Yamada et

90 al., 2013b) used only a single probability of 0.5$)$. Recent studies have extended this earlier

91 work asking whether captive macaques also distort probabilities in the same way humans

92 do (Farashahi et al., 2018; Ferrari-Toniolo et al., 2019; Nioche et al., 2021; Stauffer et al.,

93 2015), but no research has identified yet whether the activity of individual neurons in the

94 reward circuitry computes the subjective valuation of risky prospects in a way that is

95 consistent with prospect theory.

96 Thus, we targeted the reward-related cortical and subcortical structures of non-

97 human primates (Haber and Knutson, 2010): central part of the orbitofrontal cortex (cOFC,

98 area 13M), medial part of the orbitofrontal cortex (mOFC, area 14O), dorsal striatum (DS,

99 the caudate nucleus), and ventral striatum (VS). We measured the neural activity in a non-

100 choice situation while monkeys perceived a lottery with a range of probability and

101 magnitude of rewards (10 reward magnitudes by 10 reward probabilities, resulting in 100

102 unique lotteries). We found neurons whose activity can be parameterized using the

103 prospect theory model as a multiplicative combination of subjective value (utility) and

104 subjective probability (probability weighting) functions. A simple network model that

105 aggregates these subjective valuation signals via linear integration successfully 
Yamada et al.

106 reconstructed the monkey's risk preference and subjective probability perception

107 estimated from choices monkeys made in other situations. This is an evidence for a

108 neuronal prospect theory model employing distributed computations in the reward circuitry.

\section{RESULTS}

\section{Prospect theory and decision characteristics in monkeys}

112 We estimated the monkeys' subjective valuations of risky rewards using a gambling task

113 (Figure 1A) (Yamada et al., 2021) similar to those used with human subjects in economics

114 (Hey and Orme, 1994). In the choice trials, monkeys chose between two options that

115 offered an amount of liquid reward with some probability. The monkeys fixated on a central

116 gray target, and then, two options were presented visually as pie charts displayed on the

117 left and right sides of the screen. The number of green pie segments indicated the

118 magnitude of the liquid reward in $0.1 \mathrm{~mL}$ increments $(0.1-1.0 \mathrm{~mL})$, and the number of blue

119 pie segments indicated the probability of receiving the reward in 0.1 increments $(0.1-1.0$,

120 where 1.0 indicates a 100\% chance). The monkeys chose between the left and right

121 targets by fixating on one side. Following the choice, the monkeys received or did not

122 receive the amount of liquid reward associated with their chosen option according to their

123 corresponding probability. In each choice trial, two out of the 100 possible combinations of

124 probability and magnitude of rewards were randomly selected and allocated to the left- and

125 right-side target options. We used all data collected after each monkey learned to

126 associate the probability and magnitude with the pie-chart stimuli. This included 44,883

127 decisions made by monkey SUN (obtained in 884 blocks over 242 days) and 19,292

128 decisions by monkey FU (obtained in 571 blocks over 127 days). These well-trained

129 monkeys, like humans, showed behavior consistent with utility maximization, selecting on 
Yamada et al.

130 average options with the higher expected value, i.e., probability times magnitude (Figure

131 1B). In the experiment, a block of choice trials was occasionally interleaved with a block of

132 single-cue trials (Figure 1C), during which neural recordings were made. In these trials, the

133 monkey did not make a choice but passively viewed a single lottery cue, which offered

134 some amount of reward with some probability given after a delay.

135 We estimated each monkey's utility and probability weighting functions from their

136 choice behavior using the standard parametrizations in the literature. For the utility

137 function, we used the power utility function $u(m)=m^{\alpha}$, where $m$ indicates the magnitude of

138 reward, $\alpha>1$ indicates convex utility (risk-seeking behavior), $\alpha<1$ indicates concave utility

139 (risk aversion), and $\alpha=1$ indicates linear utility (risk neutrality). For the probability

140 weighting function $w(p)$, we used one-parameter, $w(p)=\exp \left(-(-\log p)^{v}\right)$, and two-

141 parameter, $w(p)=\exp \left(-\delta(-\log p)^{v}\right)$, Prelec functions. The one-parameter version is nested

142 in the two-parameter version (when $\delta=1$ ) for ease of comparison. Overall, we estimated

143 the following four models of the utility of receiving reward magnitude $m$ with probability $p$,

$144 \quad V(p, m):$

1. EV: expected value $\quad V(p, m)=p m$

146

$$
\text { 2. EU: expected utility } \quad V(p, m)=p m^{\alpha}
$$

147 3. PT1, one-parameter Prelec: prospect theory with $w(p)$ as in $(\mathrm{Wu}$ and Gonzalez, $148 \quad$ 1996)

$$
V(p, m)=\exp \left(-(-\log p)^{\gamma}\right) m^{\alpha}
$$

4. PT2, two-parameter Prelec: prospect theory with $w(p)$ as in (Prelec, 1998)

$152 \alpha, \delta$, and $\gamma$ are free parameters, and $p$ and $m$ are the probability and magnitude of reward 153 cued by the lottery, respectively. The parameters $\delta$ and $\gamma$ control the subproportionality 
Yamada et al.

154 and regressiveness of $w(p)$. We assumed that subjective probabilities and utilities are

155 integrated multiplicatively, as is customary in economic theory, yielding $V(p, m)=w(p) u(m)$.

156 The probability of the monkey choosing the lottery on the right side $\left(L_{R}\right)$ instead of the

157 lottery on the left side $\left(L_{L}\right)$ was estimated using a logistic choice function:

$$
P\left(L_{R}\right)=1 /\left(1+e^{-z}\right)
$$

159 where $z=\beta\left(V\left(L_{R}\right)-V\left(L_{L}\right)\right)$, and the free parameter $\beta$ controls the degree of stochasticity 160 observed in the choices.

161 To determine which model best describes the behavior of a monkey, we used 162 Akaike's Information Criterion (AIC), which measures the goodness of model fit with a 163 penalty for the number of free parameters employed by the model (see Methods for more 164 details). Among the four models, PT2 had the lowest AIC and outperformed EV, EU, and 165 PT1 in both monkeys (Figure 1D). In the best-fit model, the utility function was concave 166 (Figure 1E; one-sample t-test, $\alpha=0.80, \mathrm{z}=46.10, \mathrm{P}<0.001$ in monkey SUN; $\alpha=0.52, \mathrm{P}$ $167<0.001, z=25.04$ in monkey FU), indicating that monkeys were risk-averse. Notably, for 168 both monkeys, the probability weighting functions were concave instead of the inverse-S 169 shape traditionally assumed in humans (Figure $1 \mathrm{~F}$; one-sample t-test, $\delta=0.57, z=86.51$, $170 \mathrm{P}<0.001$ in monkey SUN; $\delta=0.57, \mathrm{z}=52.77, \mathrm{P}<0.001$ in monkey $\mathrm{FU} ; \gamma=1.43, \mathrm{z}=$ 171 47.29, $\mathrm{P}<0.001$ in monkey SUN; $\gamma=1.12, \mathrm{z}=25.68$ in monkey $\mathrm{FU}, \mathrm{P}<0.001)$. Overall, 172 we conclude that in monkeys, utility functions estimated from behavior are concave, similar 173 to those in humans, but monkeys distort probability differently compared to what is usually 174 assumed for human decision-makers.

175

176 Neural signals for subjective valuations are distributed in the reward circuitry 
Yamada et al.

177 We recorded single-neuron activity during the single-cue task (Figure 1C) from neurons in

178 the DS ( $\mathrm{n}=194)$, VS (144), cOFC (190), and mOFC (158) (Figure 1G). These brain regions

179 are known to be involved in decision-making. We first identified neurons whose activity

180 represents the key reward statistics - probability and magnitude - that underlie the

181 expected value, expected utility, and prospect theory. These neurons were identified by

182 regressing neural activity on probability and magnitude of rewards, and the neurons

183 included in our analysis were those that had either both positive or both negative

184 regression coefficients (See Methods).

185 An example of activity during a one-second time window after cue onset is shown in

186 Figure $1 \mathrm{H}$. This DS neuron showed an activity modulated by both the probability and

187 magnitude of rewards with positive regression coefficients $(\mathrm{P}+\mathrm{M}+$ type; probability,

188 regression coefficient, $r=13.51, t=8.57, \mathrm{P}<0.001 ;$ magnitude, $\mathrm{r}=12.27, \mathrm{t}=7.79, \mathrm{P}<$

189 0.001). Neuronal firing rates increased as the reward probability increased and as the

190 reward magnitude increased, representing a positive coding type (Figure 1H, right).

191 Similarly, some neurons showed an activity modulated by both the probability and

192 magnitude of rewards with negative regression coefficients, representing a negative

193 coding type (P-M- type). In total, these types of activity were observed in $24 \%(164 / 686)$ of

194 all recorded neurons in at least one of the four analysis epochs during the 2.5-s cue period.

195 The proportions of these signals in each brain region were different (DS, 22\%, 43/194, VS,

$19632 \%, 45 / 141$, cOFC, 31\%, 59/190, mOFC, $11 \%, 17 / 158$, chi-squared test, $X^{2}=25.59, \mathrm{df}=$

$1973, \mathrm{P}<0.001)$. These neurons were evident across the entire cue period (Figure 1I), during

198 which the monkeys perceived the probability and magnitude of rewards. 
Yamada et al.

201 For visual inspection of the potential neuronal signature of $V(p, m)$, we predicted from the

202 behavioral estimates how the observed neuronal firing rates should look like in each of the

203 four models: expected value (Figure 2A, EV), expected utility (Figure 2B, EU), and

204 prospect theory (Figure 2C and 2D, PT1 and PT2, respectively). In each of the models, the

205 neural firing rate $R$ is given by:

$$
R=g w(p) u(m)+b
$$

207 where the predicted neuronal responses $R$, the output of the model, integrates the 208 subjective value function (i.e., utility, $u(m)$ ) and subjective probability function (i.e., 209 probability weight, $w(p)) . b$ is a free parameter that captures the baseline firing rates in the 210 probability-magnitude space. $g$ determines the magnitude of the neural responses to $u(m)$ 211 and $w(p) . u(m)$ and $w(p)$ are specified for each model as described above (see the 212 formulas in Figure 2 and Methods).

213 Next, we aimed to assess which of the models best captures the neuronal discharge 214 rates in each brain region. Therefore, we fitted the activities of individual neurons with 215 each of the four models, treating $b, g, \alpha, \delta$, and $\gamma$ as free parameters. Our carefully 216 designed set of lottery stimuli - a sampling matrix of 10 rewards by 10 probabilities 217 allowed us to perform a reliable estimation of these five free parameters for each activity of 218 neurons. To determine which model best describes the observed neuronal firing rate in 219 each individual neurons, we used the AIC. As demonstrated for an example neuron in 220 Figure 3A, the activity of this DS neuron was best explained by prospect theory with a two221 parameter probability weighting function (Figure 3B, PT2). For this neuron, PT2 had the 222 smallest AIC values with the highest percentage of explained variance. The output $R$ of the 223 fitted PT2 model described the activity pattern well (Figure 3C), as well as the observed 
Yamada et al.

224 activity (Figure 3A), in which the neural utility function and subjective probability weighting

225 function were parameterized (Figure 3D) via a multiplicative relation in the model.

226 To understand which model best describes the neural activity in each brain region, we

227 determined the goodness-of-fit score for each activity of the neurons as the difference in

228 AIC between each of the models (EU, PT1, and PT2) and the EV model. Here, we treated

229 the EV model as the baseline because it is the simplest model and a predecessor of the

230 other models in the economics literature. Figure 3E shows the probability density of the

231 goodness-of-fit score differences for each brain region separately. The vertical dashed

232 lines at zero indicate no difference in the AIC of the EV model and that of the model under

233 consideration. A model that shows more deviation to the right of the graph indicates a

234 better fit.

235 Overall, prospect theory (PT2) best described the activity of most neural populations in

236 the reward circuitry (DS, VS, and cOFC), except for mOFC activity. We statistically

237 compared the AIC values among the four models. The comparisons indicated that the PT2

238 model was best at describing DS, VS, and cOFC activity as a whole (one-sample t-test

239 after subtracting models' AIC scores; DS: $d f=62, E V-E U, t=0.94, P=0.35, E U-P T 1, t=$

$2401.03, P=0.31, P T 1-P T 2, t=3.01, P=0.004 ; V S: d f=92, E V-E U, t=2.42, P=0.017, E U-$

$241 \mathrm{PT} 1, \mathrm{t}=4.00, \mathrm{P}<0.001, \mathrm{PT} 1-\mathrm{PT} 2, \mathrm{t}=3.91, \mathrm{P}<0.001 ; \mathrm{cOFC}: \mathrm{df}=115, \mathrm{EV}-\mathrm{EU}, \mathrm{t}=2.90, \mathrm{P}$

$242=0.004, \mathrm{EU}-\mathrm{PT} 1, \mathrm{t}=0.65, \mathrm{P}=0.52, \mathrm{PT} 1-\mathrm{PT} 2, \mathrm{t}=6.18, \mathrm{P}<0.001$, not shown for all).

243 However, the best descriptive model of the mOFC activity could not be determined (one-

244 sample t-test; mOFC: $d f=26, E V-E U, P=0.60, E U-P T 1, P=0.10, P T 1-P T 2, P=0.11)$,

245 suggesting that mOFC neurons simply signal expected values, without any distortions to

246 objective probability and magnitude of rewards during the perception of the lottery. 
Yamada et al.

Next, we asked whether neurons differentially encode subjective valuations based on

248 their location (DS, VS, and cOFC). For this purpose, we used the PT2 model estimates $b$,

$249 g, \alpha, \delta$, and $\gamma$ of individual activity of neurons, including both positive and negative coding

250 types. We clustered these five parameters using k-means clustering algorithms following

251 principal component analysis (PCA) across the neural population in the DS, VS, and cOFC

252 (Figure 4A and 4B, see Methods). The five predominant clusters, C1 to C5, were obtained

253 after PCA based on the four principal components (Figure 4B). These five clusters were

254 observed in similar proportion across the three brain regions with only slight differences

255 (Figure 4C). One small difference was that the VS contained a smaller proportion of the

256 predominant cluster than the other two regions (chi-squared test, $X^{2}=18.15$, df $=8, P=$

257 0.020)

258 Across the DS, VS, and cOFC, the predominant cluster, C1, represented $48 \%$ of all

259 activity (Figure 4D, top row; mean values: $b=-0.68, g=10.1, \alpha=0.64, \delta=1.30, \gamma=2.64$ ).

260 Its output, $R$, was described by a combination of a concave utility function and an S-

261 shaped probability weighting function (Figure 4D, see the third and fourth columns in the

262 top row). The second predominant cluster, C2, was also best described with a concave

263 utility function, but its probability weighting function was concave. This cluster was mostly

264 composed of neurons with negative coding of probability and magnitude of rewards

265 (Figure 4D, middle row; $b=10.6, g=-10.1, \alpha=0.29, \delta=0.38, \gamma=1.82$ ). Because the

266 coding gain was negative (Figure 4D, middle left, note that axis values are plotted from 1.0

267 to 0 ), the convex curvature (Figure 4D, left column in the middle row) of the firing rate

268 corresponds to the concave functions $u(m)$ and $p(w)$. A considerable proportion of neurons

269 (9\%), C3, showed output well described by a convex utility function and an S-shaped 
Yamada et al.

270 probability weighting function with a smaller gain compared to C1 and C2 (Figure 4D,

271 bottom; $b=2.6, g=7.2, \alpha=3.2, \delta=3.5, \gamma=2.7)$.

272 These clusters of neurons parameterized by the prospect theory model were not

273 localized and were instead found scattered across most parts of the reward circuitry (DS,

274 VS, and cOFC), suggesting that distributed coding underlies internal subjective valuations

275 under risk.

277 Reconstruction of internal preference parameters from observed neural activity in 278 monkeys

279 Lastly, we reconstructed the monkeys' internal valuations of passively viewed lotteries

280 from the observed neural activity to assess how well they match the utility and probability

281 weighting functions estimated from the behavioral choices. To do so, we constructed a

282 simple three-layered network model as a minimal rate model, a primitive version of the

283 advanced models used recently (Juslin et al., 2003; Ohshiro et al., 2011), and simulated

284 the choices of this network model (Figure 5). We assumed that outputs reflecting $V(p, m)$ in

285 each neural cluster C1 to C5 (Figure 5A, first layer) were linearly integrated by the network

286 (Figure 5A, second layer, population SEVs, see Methods). The activities in clusters 1, 3,

287 and 5 (mostly composed of $\mathrm{P}+\mathrm{M}+$ neurons) were linearly summed, and the activities in

288 clusters 2 and 4 (mostly composed of P-M- neurons) were subtracted to integrate the

289 opposed signals (hence, linear summation of an inversed signal). To simulate choice, we

290 generated two identical population SEVs for the left $\left(\Sigma R_{L}\right)$ and right $\left(\Sigma R_{R}\right)$ target options

291 and used a random utility model for selecting one option (Figure 5A, third layer, sigmoid

292 choice function). Overall, we simulated 40,000 choices - four times each possible

293 combination of 100 lotteries, $L(p, m)$. 
Yamada et al.

294 While our network model used neural signals modeled by prospect theory during 295 passive viewing, these simulated choice patterns based on the clustered neuronal 296 prospect theory model were very similar to the actual gambling behaviors of the monkeys

297 (Figures 5B and 1B). When estimating the utility function and probability weighting function

298 of these simulated choices, we observed concave utility functions and concave probability

299 weighting functions similar to those obtained from the actual gambling behavior (Figure

$3005 \mathrm{C}$ ). Thus, we conclude that a distributed neural code that accumulates individual neuronal

301 signals can explain the internal subjective valuations of monkeys.

303 Discussion

304 Prospect theory is the dominant theory of choice in behavioral economics, but it remains 305 elusive whether the theory is only descriptive of human behavior or has a deeper meaning 306 in the sense that it also describes an underlying neuronal computation that extends to 307 other species. Previous human neuroimaging studies have demonstrated that neural 308 responses to rewards measured through blood oxygen levels can be described using 309 prospect theory (Hsu et al., 2009; Tobler et al., 2008; Tom et al., 2007) but with limited 310 resolution in temporal and spatial domains. Here, we provided the first evidence that the 311 activity of individual neurons in the reward circuitry (DS, VS, and cOFC) of monkeys 312 perceiving a lottery can be captured based on the prospect theory model as a 313 multiplicative combination of utility and probability weighting functions (Figure 4). One 314 pivotal question is how these various subjective preference signals are transformed into 315 behavioral choices through information processing via neural networks. Our clustering 316 analysis of the parameterized neuronal activity revealed that these signals were similarly 317 distributed across the VS, DS, and cOFC (Figure 4C). Our minimal rate model of a three- 
Yamada et al.

318 layered network successfully reconstructed the internal valuation of risky rewards

319 observed in monkeys (Figure 5), suggesting that these subjective valuation signals in the

320 reward circuitry are integrated into the brain to construct a decision output from risky

321 perspectives.

322 Previous studies have shown that neuronal signals related to cognitive and motor

323 functions are widely observed in many brain regions (Bouton et al., 2018; Coghill, 2020;

324 Nestor et al., 2011; Pinel et al., 2004; Simon et al., 2006; Stefanini et al., 2020; Wixted et

325 al., 2014). These distributed neuronal signals suggest that a distributed neural code is a

326 common computation in the brain. The recent development of large-scale neural recording

327 technologies verified that this is a common computational mode (Steinmetz et al., 2019);

328 the analysis of approximately 30,000 neurons in 42 regions of the rodent brain revealed

329 that behaviorally relevant task parameters are observed throughout the brain. Our results

330 from the reward-related brain regions are in line with this view, except for the mOFC,

331 where fewer encodings of probability and magnitude of rewards were observed (Figures 1 I

332 and $3 \mathrm{E})$. This might be because the medial-lateral axis in the reward circuitry yields a

333 significant difference in reward-based decision-making (Haber and Knutson, 2010). The

334 distributed code may require some input-output functions (Vankov and Bowers, 2017) to

335 process the probability and magnitude of rewards and integrate these information to

336 estimate the expected subjective utility, at least in some neural populations. One possible

337 information processing for this input-output mapping can be achieved by neural population

338 dynamics (Chen and Stuphorn, 2015; Gardner et al., 2019; Yoo and Hayden, 2020), in

339 which some subclusters of neurons can process information moment-by-moment as a

340 dynamical system. Stable neural population dynamics in the VS and cOFC were indeed 
Yamada et al.

341 observed in contrast to the fluctuating signals in the DS population (Yamada et al., 2021),

342 which may reflect some differences in distributed coding.

343 One limitation of our study is that our application of prospect theory is limited to the

344 domain of gains, since unlike in human studies that use money as the reward, it is

345 impossible to take fluid rewards from monkeys to make them experience losses.

346 Nevertheless, our study adds important behavioral evidence to the growing literature on

347 prospect theory preferences in primates. Recent studies of captive macaques have begun

348 to investigate distortions in the perception of probabilities, with inconsistent results across

349 studies (Eisenreich et al., 2019; Farashahi et al., 2018; Ferrari-Toniolo et al., 2019; Nioche

350 et al., 2019; Nioche et al., 2021; Stauffer et al., 2015). The probability weighting function

351 was inverse S-shaped (Farashahi et al., 2018; Ferrari-Toniolo et al., 2019), S-shaped

352 (Nioche et al., 2021; Stauffer et al., 2015), or concave (Ferrari-Toniolo et al., 2021; Ferrari-

353 Toniolo et al., 2019). Although we consistently found that the probability weighting

354 functions of our two well-trained monkeys were concave, most studies conducted in

355 humans have found inverse-S-shaped probability weighting functions at the aggregate

356 level, with a large amount of heterogeneity at the individual level (Abdellaoui, 2000; Bruhin

357 et al., 2010; Fehr-Duda et al., 2011; Harbaugh et al., 2002; Harrison and Rutstrom, 2009;

358 Hsu et al., 2009; Tobler et al., 2008) indicating an inconsistency across the two species.

359 Furthermore, the monkeys in the present study had concave utility functions while most

360 previous studies have found that monkeys have a convex (Farashahi et al., 2018; Stauffer

361 et al., 2015) or concave (Eisenreich et al., 2019; Ferrari-Toniolo et al., 2021; Nioche et al.,

362 2019; Yamada et al., 2013b) utility over rewards in the gain domain. In conclusion, our

363 monkeys had concave utility functions, similar to our previous findings in monkeys 
bioRxiv preprint doi: https://doi.org/10.1101/2021.12.18.473272; this version posted December 20, 2021. The copyright holder for this preprint

(which was not certified by peer review) is the author/funder, who has granted bioRxiv a license to display the preprint in perpetuity. It is made available under aCC-BY-NC-ND 4.0 International license.

Yamada et al.

364 (Yamada et al., 2018; Yamada et al., 2013b) as well as in humans. But unlike humans, our

365 monkeys had concave probability weighting functions.

366 Summing up, we provided novel evidence that the activity of the individual neurons in

367 the reward circuitry can be described using prospect theory and that the probability

368 distortions estimated from the monkeys' behaviors are different than those usually

369 assumed for humans.

370 
Yamada et al.

\section{METHODS}

\section{Subjects and experimental procedures}

373 Two rhesus monkeys performed the task (Macaca mulatta, SUN, $7.1 \mathrm{~kg}$, male; Macaca

374 fuscata, FU, $6.7 \mathrm{~kg}$, female). All experimental procedures were approved by the Animal

375 Care and Use Committee of the University of Tsukuba (Protocol No H30.336) and

376 performed in compliance with the US Public Health Service's Guide for the Care and Use

377 of Laboratory Animals. Each animal was implanted with a head-restraint prosthesis. Eye

378 movements were measured using a video camera at $120 \mathrm{~Hz}$. Visual stimuli were

379 generated by a liquid-crystal display at $60 \mathrm{~Hz}$, placed $38 \mathrm{~cm}$ from the monkey's face when

380 seated. The subjects performed the cued lottery task five days a week. The subjects

381 practiced the cued lottery task for 10 months, after which they became proficient in

382 choosing lottery options.

384 Cued lottery tasks

385 Animals performed one of two visually cued lottery tasks: a single-cue task or a choice 386 task.

\section{Single-cue task}

389 At the beginning of each trial, the monkeys had $2 \mathrm{~s}$ to align their gaze within $3^{\circ}$ to a $1^{\circ}$ -

390 diameter gray central fixation target. After fixing for $1 \mathrm{~s}$, an $8^{\circ}$ pie chart providing

391 information about the probability and magnitude of rewards was presented for $2.5 \mathrm{~s}$ at the

392 same location as the central fixation target. Probability and magnitude were indicated by

393 the numbers of blue and green pie chart segments, respectively. The pie chart was then

394 removed and $0.2 \mathrm{~s}$ later, a $1-\mathrm{kHz}$ and $0.1-\mathrm{kHz}$ tone of $0.15-\mathrm{s}$ duration indicated reward and 
Yamada et al.

395 no-reward outcomes, respectively. The high tone preceded reward delivery by $0.2 \mathrm{~s}$,

396 whereas the low tone indicated that no reward was delivered. The animals received a

397 liquid reward, as indicated by the number of green pie chart segments with the probability

398 indicated by the number of blue pie chart segments. An intertrial interval of 4-6 s followed

399 each trial.

$401 \quad$ Choice task

402 At the beginning of each trial, the monkeys had $2 \mathrm{~s}$ to align their gaze within $3^{\circ}$ to a $1^{\circ}$ 403 diameter gray central fixation target. After fixation for $1 \mathrm{~s}$, two peripheral $8^{\circ}$ pie charts 404 providing information about the probability and magnitude of rewards for each of the two 405 target options were presented for $2.5 \mathrm{~s}$ at $8^{\circ}$ to the left and right of the central fixation 406 location. The gray $1^{\circ}$ chosen targets appeared at the same locations. After a 0.5-s delay, 407 the fixation target disappeared, cueing saccade initiation. The monkeys were allowed $2 \mathrm{~s}$ 408 to make their choice by shifting their gaze to either target within $3^{\circ}$ of the chosen target. A $4091-\mathrm{kHz}$ and $0.1-\mathrm{kHz}$ tone sounded for $0.15 \mathrm{~s}$ to denote reward and no-reward outcomes, 410 respectively. The animals received a liquid reward, as indicated by the number of green 411 pie chart segments of the chosen target with the probability indicated by the number of 412 blue pie chart segments. An intertrial interval of 4-6 s followed each trial.

\section{Payoff, block structure, and data collection}

415 Green and blue pie charts respectively indicated reward magnitudes from 0.1 to $1.0 \mathrm{~mL}$, in $4160.1 \mathrm{~mL}$ increments, and reward probabilities from 0.1 to 1.0 , in 0.1 increments. A total of 417100 pie chart combinations were used. In the single-cue task, each pie chart was 418 presented once in a random order, allowing monkeys to experience all 100 lotteries within 
Yamada et al.

419 a certain period. In the choice task, two pie charts were randomly allocated to the left and

420 right targets in each trial. Approximately 30-60 trial blocks of the choice task were

421 sometimes interleaved with 100-120 trial blocks of the single-cue task.

\section{Calibration of the reward supply system}

424 A precise amount of liquid reward was delivered to the monkeys using a solenoid valve.

425 An 18-gauge tube $(0.9 \mathrm{~mm}$ inner diameter) was attached to the tip of the delivery tube to 426 reduce the variation across trials. The amount of reward in each payoff condition was 427 calibrated by measuring the weight of water with $0.002 \mathrm{~g}$ precision $(2 \mu \mathrm{L})$ on a single-trial 428 basis. This calibration method was the same as that used in (Yamada et al., 2018).

$430 \quad$ Electrophysiological recordings

431 We used conventional techniques to record single-neuron activity from the DS, VS, cOFC,

432 and mOFC. Monkeys were implanted with recording chambers $(28 \times 32 \mathrm{~mm})$ targeting the 433 OFC and striatum, centered $28 \mathrm{~mm}$ anterior to the stereotaxic coordinates. The locations 434 of the chambers were verified using anatomical magnetic resonance imaging. We used a 435 tungsten microelectrode (1-3 $\mathrm{M} \Omega, \mathrm{FHC})$ to record the neurons. Electrophysiological 436 signals were amplified, band-pass-filtered, and monitored. Single-neuron activity was 437 isolated based on the spike waveforms. We recorded from the four brain regions of a 438 single hemisphere of each of the two monkeys: 194 DS neurons (98 and 96 from monkeys 439 SUN and FU, respectively), 144 VS neurons (89, SUN and 55, FU), 190 cOFC neurons 440 (98, SUN and 92, FU), and 158 mOFC neurons (64, SUN and 94, FU). The activity of all 441 single neurons was sampled when the activity of an isolated neuron demonstrated a good 442 signal-to-noise ratio (> 2.5). Blinding was not performed. The sample sizes required to 
Yamada et al.

443 detect effect sizes (the number of recorded neurons, the number of recorded trials in a

444 single neuron, and the number of monkeys) were estimated in reference to previous

445 studies (Chen and Stuphorn, 2015; Yamada et al., 2013a; Yamada et al., 2018). Neural

446 activity was recorded during 100-120 trials of the single-cue task. During the choice trials,

447 the neural activity was not recorded. Presumed projection neurons (phasically active

448 neurons, (Yamada et al., 2016)) were recorded from the DS and VS, whereas presumed

449 cholinergic interneurons (tonically active neurons, (Inokawa et al., 2020; Yamada et al., 450 2004) were not recorded.

\section{Statistical analysis}

453 For statistical analysis, we used the statistical software R and Stata. All statistical tests

454 were two-tailed. We used standard maximum likelihood procedures to estimate utility 455 functions and probability weighting functions in Stata. We performed a neural analysis and 456 simulation to reconstruct the choice from a neural model in $\mathrm{R}$.

\section{Behavioral analysis}

459 We first examined whether the choice behavior of a monkey depended on the expected

460 values of the two options located on the left and right sides of the screen. We pooled 461 choice data across all recording sessions (monkey SUN, 884 sessions, 242 days; monkey 462 FU, 571 sessions, 127 days), yielding 44,883 and 19,292 choice trials for monkeys SUN 463 and FU, respectively. The percentage of the right target choices was estimated from the 464 pooled choice data for all combinations of the expected values of the left and right target 465 options. This result has been reported previously (Yamada et al., 2021). 
Yamada et al.

\section{Economic models}

468 We estimated the parameters of the utility and probability weighting functions within a 469 random utility framework. Specifically, a lottery $L(p, m)$ denoted a gamble that pays $m$ 470 (magnitude of the offered reward in $\mathrm{mL}$ ) with a probability $p$ or 0 otherwise. We assumed a 471 popular constant relative risk attitude (CRRA, also known as power utility function), $u(m)=$ $472 \mathrm{~m}^{\alpha}$, and considered the previously proposed probability weighting functions. We assumed 473 two subjective probability functions $w(p)$ commonly used in the prospect theory; one474 parameter Prelec (PT1): $w(p)=\exp \left(-(-\log p)^{\gamma}\right)(\mathrm{Wu}$ and Gonzalez, 1996) and two475 parameter Prelec (PT2): $w(p)=\exp \left(-\delta(-\log p)^{v}\right)$ (Prelec, 1998). We assumed that 476 subjective probabilities and utilities are integrated multiplicatively per standard economic 477 theory, yielding the expected subjective utility function $V(p, m)=w(p) u(m)$.

478 The probability of a monkey choosing the lottery on the right side $\left(L_{R}\right)$ instead of the 479 lottery on the left side $\left(L_{L}\right)$ was estimated using a logistic choice function:

$$
P\left(L_{R}\right)=1 /\left(1+e^{-z}\right)
$$

481 where $z=\beta\left(V\left(L_{R}\right)-V\left(L_{L}\right)\right)$, and the free parameter $\beta$ controls the degree of stochasticity 482 observed in the choices. We fitted the data by maximizing log-likelihood and choosing the 483 best structural model to describe the monkeys' behavior using the AIC (Burnham and 484 Anderson, 2004).

$$
\mathrm{AlC}_{\text {Model }}=-2 L+2 k
$$

486 where $L$ is the maximum log-likelihood of the model, and $k$ is the number of free 487 parameters.

488 In each fitted model, whether $\alpha, \delta$, and $\gamma$ were significantly different from zero was 489 determined using a one-sample t-test at $\mathrm{P}<0.05$. Whether $\alpha, \delta$, and $\gamma$ were significantly 490 different from one was also determined using a one-sample t-test at $\mathrm{P}<0.05$. 
Yamada et al.

\section{Neural analysis}

\section{Basic firing properties}

494 Peristimulus time histograms were drawn for each single-neuron activity aligned at the 495 onset of a visual cue. The average activity curves were smoothed using a 50-ms Gaussian 496 kernel $(\sigma=50 \mathrm{~ms})$. Basic firing properties, such as peak firing rates, peak latency, and 497 duration of peak activity (half peak width), were compared among the four brain regions 498 using parametric or nonparametric tests, with a statistical significance level of $P<0.05$.

499 Baseline firing rates during $1 \mathrm{~s}$ before the appearance of central fixation targets were also 500 compared with a statistical significance level of $\mathrm{P}<0.05$. These basic firing properties 501 have been described in Yamada et al., 2021.

502 We analyzed neural activity during a 2.5-s period during pie chart stimulus 503 presentation in the single-cue task. We estimated the firing rates of each neuron during the 504 1-s time window every $0.5 \mathrm{~s}$ after the onset of the cue stimuli. No Gaussian kernel was 505 used.

\section{Pre-screening neural activity for economic model fits}

508 To determine which neurons were sensitive to the probability and magnitude cued by a 509 lottery, without assuming any specific model, neural discharge rates $(F)$ were regressed on

510 a linear combination of a constant and the probability and magnitude of rewards:

$$
F=b_{0}+b_{p} p+b_{m} m
$$

512 where $p$ and $m$ are the probability and magnitude of the rewards indicated by the pie chart, 513 respectively. $b_{0}$ is the intercept. If $b_{p}$ and $b_{m}$ were not 0 at $\mathrm{P}<0.05$, the discharge rates 514 were regarded as significantly modulated by that variable. 
Yamada et al.

$515 \quad$ Based on the linear regression, two types of neural modulations were identified: the

516 "P+M+" type with a significant $b_{p}$ and a significant $b_{m}$ both having a positive sign (i.e.,

517 positive $b_{\mathrm{p}}$ and positive $b_{\mathrm{m}}$ ) and the "P-M-" type with a significant $b_{\mathrm{p}}$ and a significant $b_{\mathrm{m}}$

518 both having a negative sign (i.e., negative $b_{p}$ and negative $b_{m}$ ). Both types of the neuronal

519 signal could represent the economic decision statistics described in the next section.

$521 \quad$ Neural economic models

522 We fitted the four neural models of subjective valuation of lottery $L(p, m)$ to the activity of

523 the pre-selected neurons that were sensitive to the information of probability and 524 magnitude of rewards. The unified formula for all models is $R=g w(p) u(m)+b$, where 525 output of the model $R$ represents firing rates as a function of the subjective probability $w(p)$ 526 times the utility of reward $u(m)$, which is the subjective expected value (SEV) of a lottery 527 that reflects the monkey's lottery valuation. For neural representation of $V(p, m)$ as 528 described in the main text, we call this value function to differ from behavioral measures. In 529 all models, $g$ (magnitude of the neural response), $b$ (baseline firing rate), $\alpha$ (utility 530 curvature), $\gamma$, and $\delta$ (probability weighting) are free parameters.

531 1. Expected value model (EV).

$$
R=g p m+b
$$

533 2. Expected utility model (EU).

$$
R=g p m^{\alpha}+b
$$

535 3. Prospect theory model with one-parameter Prelec (PT1).

$$
R=g \exp \left(-(-\log p)^{\gamma}\right) m^{\alpha}+b
$$

$$
R=g \exp \left(-\delta(-\log p)^{v}\right) m^{a}+b
$$


Yamada et al.

539 To identify the structural models that best describe the activity of neurons in each brain

540 region, we fitted each of the models to the $\mathrm{P}+\mathrm{M}+$ and $\mathrm{P}-\mathrm{M}$ - type activity of each neuron on

541 a trial-by-trial basis. We estimated the combination of best-fit parameters using the $\mathrm{R}$

542 statistical software package. We used the nls() function in $\mathrm{R}$ with random initial values

543 (repeated 100 times) to find a set of parameters that minimizes nonlinear least squared

544 values.

545 For each of the four brain regions, the best-fit model showing minimal AIC was 546 selected by comparing the AIC values among the models. If the differences in AIC values

547 against the three other models were significantly different from zero in the one-sample t548 test at $\mathrm{P}<0.05$, the model was defined as the best model. For visual presentation, we 549 plotted AIC differences in comparison to the EV model as the baseline model in the 550 economics literature.

552 Construction of the neural prospect theory model

553 The estimated parameters in the best-fit model of the neuronal activity were classified 554 using PCA followed by the k-means clustering algorithm. PCA was applied once to all 555 parameters estimated in the best-fit model PT2, i.e., $g, b, \alpha, \gamma$, and $\delta$ in DS, VS, and cOFC.

556 The k-means algorithm was used to classify five types of neural responses according to 557 the PC1 to PC4 scores since the first four PCs explained more than $90 \%$ of the variance. 558 Following the classification, we define each type of cluster with the mean of each 559 estimated parameter, as the five clusters were observed in each of the DS, VS, and cOFC 560 neural populations.

561

562 Evaluation of neural model performance using simulated data 
Yamada et al.

563 We constructed a simple layered network model for simulations (Juslin et al., 2003;

564 Ohshiro et al., 2011). We simply reconstructed a neural prospect theory model from the

565 clusters above by adding each response $R$ of the five clusters. For clusters 1 , 3, and 5 , we

566 linearly summed them, while for clusters 2 and 4, which were mostly composed of P-M-

567 types, we inversed their activity by subtraction. This population SEV was filtered by a

568 ReLU (Rectified Linear Unit) function, since it mimics the firing rate. The linear sum of the

569 five clusters was allocated to the left and right target options to perform a simulation based

570 on the difference of these integrated responses. We then simulated the choice for lotteries

571 consisting of four times of all possible combinations of lotteries $L(p, m)$ using the logistic

572 function

$$
P\left(L_{R}\right)=1 /\left(1+e^{-z}\right)
$$

574 where $z=\beta\left(V\left(L_{R}\right)-V\left(L_{L}\right)\right)$ and $\beta$ is assumed to be one, i.e., no beta term. These simulated

575 choice data, composed of 40,000 choice trials, were visualized and evaluated by applying

576 the best-fit model to estimate the preference parameters $\alpha, \gamma$, and $\delta$ in $u(m)=m^{\alpha}$ and $w(p)$

$577=\exp \left(-\delta(-\log p)^{\gamma}\right)$, as well as $\beta$ in the choice function, similar to the model fit to the actual

578 behavior of the monkey. 
Yamada et al.

\section{REFERENCES}

581 Abdellaoui, M. (2000). Parameter-Free Elicitation of Utility and Probability Weighting

582 Functions. . Management Science 46, 1497-1512.

583 Abler, B., Walter, H., Erk, S., Kammerer, H., and Spitzer, M. (2006). Prediction error as a linear

584 function of reward probability is coded in human nucleus accumbens. Neuroimage 31, 790-795.

585 Berns, G.S., Capra, C.M., Chappelow, J., Moore, S., and Noussair, C. (2008). Nonlinear

586 neurobiological probability weighting functions for aversive outcomes. Neuroimage 39, 2047-

5872057.

588 Bouton, S., Chambon, V., Tyrand, R., Guggisberg, A.G., Seeck, M., Karkar, S., van de Ville, D.,

589 and Giraud, A.L. (2018). Focal versus distributed temporal cortex activity for speech sound 590 category assignment. Proc Natl Acad Sci U S A 115, E1299-E1308.

591 Bruhin, A., Fehr-Duda, H., and Epper, T. (2010). Risk and Rationality: Uncovering 592 Heterogeneity in Probability Distortion. Econometrica 78, 1375-1412.

593 Burnham, K., and Anderson, D. (2004). Multimodel inference: understanding AIC and BIC in 594 model selection. Sociol Method Res 33, 261-304.

595 Camerer, C., Loewenstein, G., and Prelec, G. (2005). Neuroeconomics: How Neuroscience Can 596 Inform Economics Journal of Economic Literature 43, 9-64.

597 Chen, X., and Stuphorn, V. (2015). Sequential selection of economic good and action in medial 598 frontal cortex of macaques during value-based decisions. Elife 4.

599 Coghill, R.C. (2020). The Distributed Nociceptive System: A Framework for Understanding

600 Pain. Trends Neurosci 43, 780-794.

601 Eisenreich, B.R., Hayden, B.Y., and Zimmermann, J. (2019). Macaques are risk-averse in a 602 freely moving foraging task. Sci Rep 9, 15091.

603 Farashahi, S., Azab, H., Hayden, B., and Soltani, A. (2018). On the Flexibility of Basic Risk 604 Attitudes in Monkeys. J Neurosci 38, 4383-4398. 
Yamada et al.

605 Fehr-Duda, H., Epper, T., Bruhin, A., and Schubert, R. (2011). Risk and rationality: The effects

606 of mood and decision rules on probability weighting. Journal of Economic Behavior and

607 Organization 78, 14-24.

608 Ferrari-Toniolo, S., Bujold, P.M., Grabenhorst, F., Baez-Mendoza, R., and Schultz, W. (2021).

609 Non-human primates satisfy utility maximization in compliance with the continuity axiom of

610 Expected Utility Theory. J Neurosci.

611 Ferrari-Toniolo, S., Bujold, P.M., and Schultz, W. (2019). Probability Distortion Depends on

612 Choice Sequence in Rhesus Monkeys. J Neurosci 39, 2915-2929.

613 Gardner, M.P.H., Conroy, J.C., Sanchez, D.C., Zhou, J., and Schoenbaum, G. (2019). Real-Time

614 Value Integration during Economic Choice Is Regulated by Orbitofrontal Cortex. Curr Biol 29,

$615 \quad 4315-4322$ e 4314.

616 Glimcher, P.W., Camerer, C.F., Fehr, E., and Poldrack, R.A. (2008). Neuroeconomics: Decision

617 Making and the Brain (New York: Elsevier).

618 Haber, S.N., and Knutson, B. (2010). The reward circuit: linking primate anatomy and human

619 imaging. Neuropsychopharmacology 35, 4-26.

620 Harbaugh, W., Krause, K., and Vesterlund, L. (2002). Risk attitudes of children and adults:

621 Choices over small and large probability gains and losses. Experimental Economics 5, 53-84.

622 Harrison, G.W., and Rutstrom, E.E. (2009). Expected utility theory and prospect theory: One

623 wedding and a decent funeral. Experimental Economics 12, 133-158.

624 Hey, J.D., and Orme, C. (1994). Investigating Generalizations of Expected Utility Theory Using

625 Experimental Data. Econometrica 62, 1291-1326.

626 Hsu, M., Krajbich, I., Zhao, C., and Camerer, C.F. (2009). Neural response to reward 627 anticipation under risk is nonlinear in probabilities. J Neurosci 29, 2231-2237.

628 Inokawa, H., Matsumoto, N., Kimura, M., and Yamada, H. (2020). Tonically Active Neurons in 629 the Monkey Dorsal Striatum Signal Outcome Feedback during Trial-and-error Search Behavior. 
Yamada et al.

630 Neuroscience 446, 271-284.

631 Juslin, P., Olsson, H., and Olsson, A.C. (2003). Exemplar effects in categorization and multiple-

632 cue judgment. J Exp Psychol Gen 132, 133-156.

633 Kahneman, D., and Tversky, A. (1979). Prospect theory: An analysis of decisions under risk.

634 Econometrica 47, 313-327.

635 Montague, P.R., Dayan, P., and Sejnowski, T.J. (1996). A framework for mesencephalic

636 dopamine systems based on predictive Hebbian learning. J Neurosci 16, 1936-1947.

637 Nestor, A., Plaut, D.C., and Behrmann, M. (2011). Unraveling the distributed neural code of

638 facial identity through spatiotemporal pattern analysis. Proc Natl Acad Sci U S A 108, 9998-

63910003.

640 Nioche, A., Bourgeois-Gironde, S., and Boraud, T. (2019). An asymmetry of treatment between

641 lotteries involving gains and losses in rhesus monkeys. Sci Rep 9, 10441.

642 Nioche, A., Rougier, N.P., Deffains, M., Bourgeois-Gironde, S., Ballesta, S., and Boraud, T.

643 (2021). The adaptive value of probability distortion and risk-seeking in macaques' decision-

644 making. Philos Trans R Soc Lond B Biol Sci 376, 20190668.

645 Ohshiro, T., Angelaki, D.E., and DeAngelis, G.C. (2011). A normalization model of multisensory 646 integration. Nat Neurosci 14, 775-782.

647 Pinel, P., Piazza, M., Le Bihan, D., and Dehaene, S. (2004). Distributed and overlapping 648 cerebral representations of number, size, and luminance during comparative judgments.

649 Neuron 41, 983-993.

650 Prelec, D. (1998). The Probability Weighting Function. Econometrica 66, 497-527.

651 Preuschoff, K., Bossaerts, P., and Quartz, S.R. (2006). Neural differentiation of expected reward 652 and risk in human subcortical structures. Neuron 51, 381-390.

653 Schultz, W., Dayan, P., and Montague, P.R. (1997). A neural substrate of prediction and reward. 654 Science 275, 1593-1599. 
Yamada et al.

655 Simon, S.A., de Araujo, I.E., Gutierrez, R., and Nicolelis, M.A. (2006). The neural mechanisms 656 of gustation: a distributed processing code. Nat Rev Neurosci 7, 890-901.

657 Stauffer, W.R., Lak, A., Bossaerts, P., and Schultz, W. (2015). Economic choices reveal 658 probability distortion in macaque monkeys. J Neurosci 35, 3146-3154.

659 Stefanini, F., Kushnir, L., Jimenez, J.C., Jennings, J.H., Woods, N.I., Stuber, G.D., Kheirbek, 660 M.A., Hen, R., and Fusi, S. (2020). A Distributed Neural Code in the Dentate Gyrus and in CA1. 661 Neuron 107, 703-716 e704.

662 Steinmetz, N.A., Zatka-Haas, P., Carandini, M., and Harris, K.D. (2019). Distributed coding of 663 choice, action and engagement across the mouse brain. Nature 576, 266-273.

664 Tobler, P.N., Christopoulos, G.I., O'Doherty, J.P., Dolan, R.J., and Schultz, W. (2008). Neuronal 665 distortions of reward probability without choice. J Neurosci 28, 11703-11711.

666 Tom, S.M., Fox, C.R., Trepel, C., and Poldrack, R.A. (2007). The neural basis of loss aversion in 667 decision-making under risk. Science 315, 515-518.

668 Vankov, I.I., and Bowers, J.S. (2017). Do arbitrary input-output mappings in parallel 669 distributed processing networks require localist coding? Language, Cognition and Neuroscience $670 \quad 32,392-399$.

671 Wixted, J.T., Squire, L.R., Jang, Y., Papesh, M.H., Goldinger, S.D., Kuhn, J.R., Smith, K.A., 672 Treiman, D.M., and Steinmetz, P.N. (2014). Sparse and distributed coding of episodic memory 673 in neurons of the human hippocampus. Proc Natl Acad Sci U S A 111, 9621-9626.

674 Wu, G., and Gonzalez, R. (1996). Curvature of the Probability Weighting Function. 675 Management Science 42, 1676-1690.

676 Yamada, H., Imaizumi, Y., and Matsumoto, M. (2021). Neural Population Dynamics Underlying 677 Expected Value Computation. J Neurosci 41, 1684-1698.

678 Yamada, H., Inokawa, H., Hori, Y., Pan, X., Matsuzaki, R., Nakamura, K., Samejima, K., 679 Shidara, M., Kimura, M., Sakagami, M., and Minamimoto, T. (2016). Characteristics of fast- 
Yamada et al.

680 spiking neurons in the striatum of behaving monkeys. Neurosci Res 105, 2-18.

681 Yamada, H., Inokawa, H., Matsumoto, N., Ueda, Y., Enomoto, K., and Kimura, M. (2013a).

682 Coding of the long-term value of multiple future rewards in the primate striatum. J

683 Neurophysiol 109, 1140-1151.

684 Yamada, H., Louie, K., Tymula, A., and Glimcher, P.W. (2018). Free choice shapes normalized

685 value signals in medial orbitofrontal cortex. Nat Commun 9, 162.

686 Yamada, H., Matsumoto, N., and Kimura, M. (2004). Tonically active neurons in the primate

687 caudate nucleus and putamen differentially encode instructed motivational outcomes of action.

688 J Neurosci 24, 3500-3510.

689 Yamada, H., Tymula, A., Louie, K., and Glimcher, P.W. (2013b). Thirst-dependent risk

690 preferences in monkeys identify a primitive form of wealth. Proc Natl Acad Sci U S A 110,

$691 \quad 15788-15793$.

692 Yoo, S.B.M., and Hayden, B.Y. (2020). The Transition from Evaluation to Selection Involves

693 Neural Subspace Reorganization in Core Reward Regions. Neuron 105, 712-724 e714. 
Yamada et al.

\section{Figure Legends}
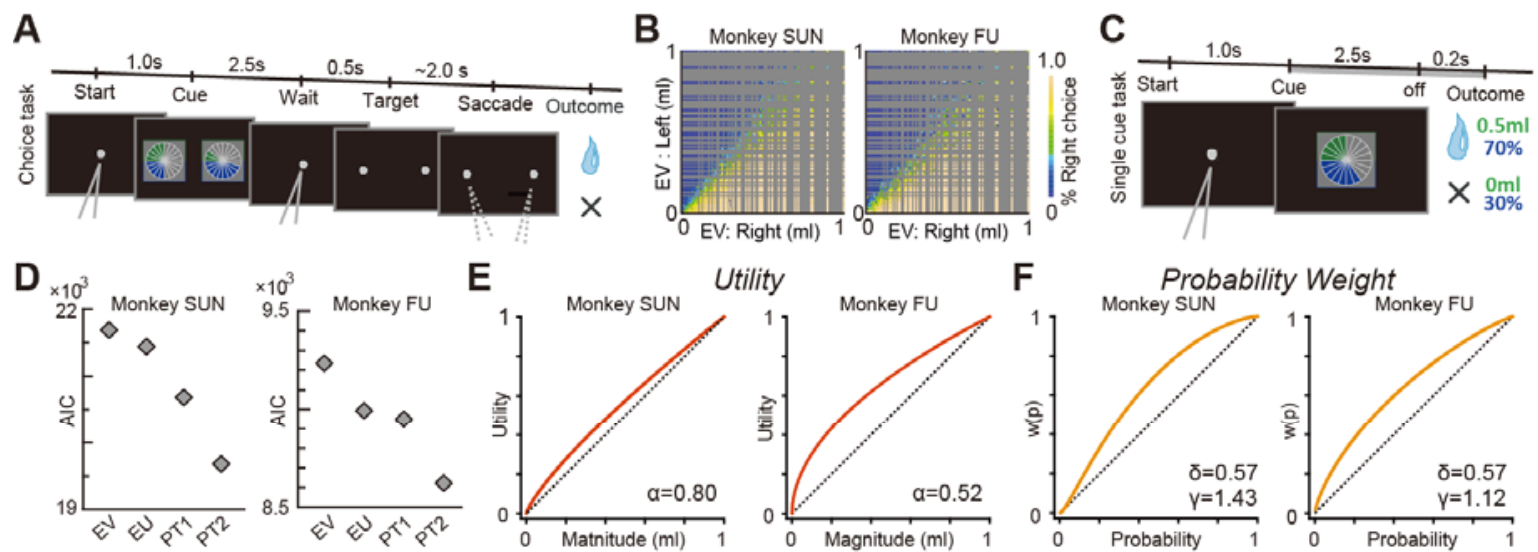

Utility
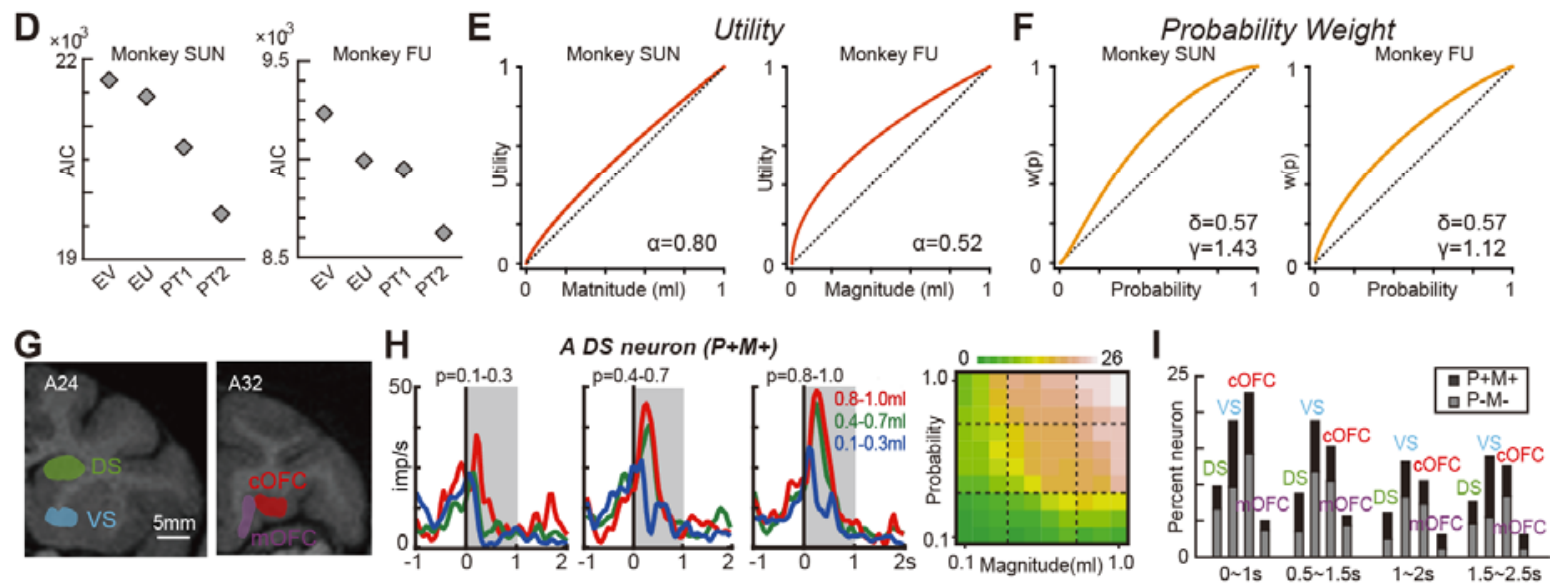

696

697

Figure 1. Cued lottery task, monkeys' choice behavior, and neural coding of probability and magnitude of rewards

699 (A) A sequence of events in the choice trials. Two pie charts representing the available

700 options were presented to the monkeys on the left and right sides of the screen. Monkeys

701 chose either of the targets by fixating on the side where it appeared.

702 (B) The frequency with which the target on the right side was selected for the expected

703 values of the left and right target options.

704 (C) A sequence of events in the single-cue trials.

705 (D) AIC values are estimated based on the four standard economic models to describe

706 monkey's choice behavior: EV, EU, PT1, and PT2. See Methods for details.

707 (E) Estimated utility functions in the best-fit model PT2.

708 (F) Estimated probability weighting functions in the best-fit model PT2.

709 (G) An illustration of neural recording areas based on coronal magnetic resonance images. 
Yamada et al.

710 (H) Example activity histogram of a DS neuron modulated by the probability and

711 magnitude of rewards with positive regression coefficients during the single-cue task. The

712 activity aligned to the cue onset is represented for three different levels of probability $(0.1-$

$7130.3,0.4-0.7,0.8-1.0)$ and magnitude $(0.1-0.3 \mathrm{~mL}, 0.4-0.7 \mathrm{~mL}, 0.8-1.0 \mathrm{~mL})$ of rewards.

714 Gray hatched time windows indicate the 1-s time window used to estimate the neural firing

715 rates shown in the right graph displaying the average smoothing between neighboring 716 pixels.

717 (I) Percentage of neurons modulated by probability and magnitude of rewards in the four 718 core reward brain regions. Black indicates activity showing positive regression coefficients 719 for probability and magnitude of rewards ( $\mathrm{P}+\mathrm{M}+$ type). Gray indicates activity showing the 720 negative regression coefficients for probability and magnitude ( $P-M-$ type).

$721(\mathrm{~A})-(\mathrm{C})$ and $(\mathrm{G})$ have been previously published in Yamada et al., 2021. 
Yamada et al.

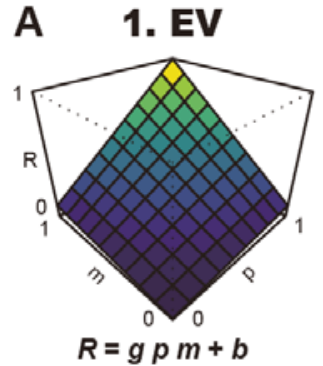

B

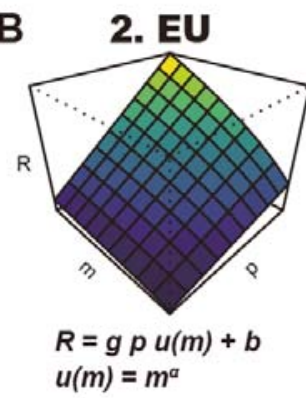

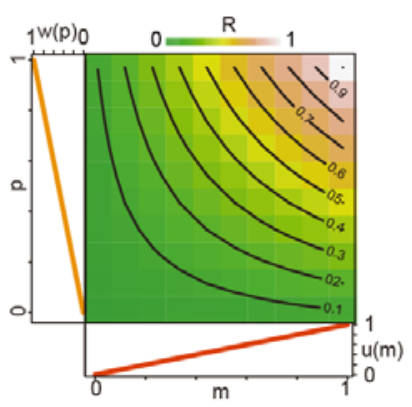
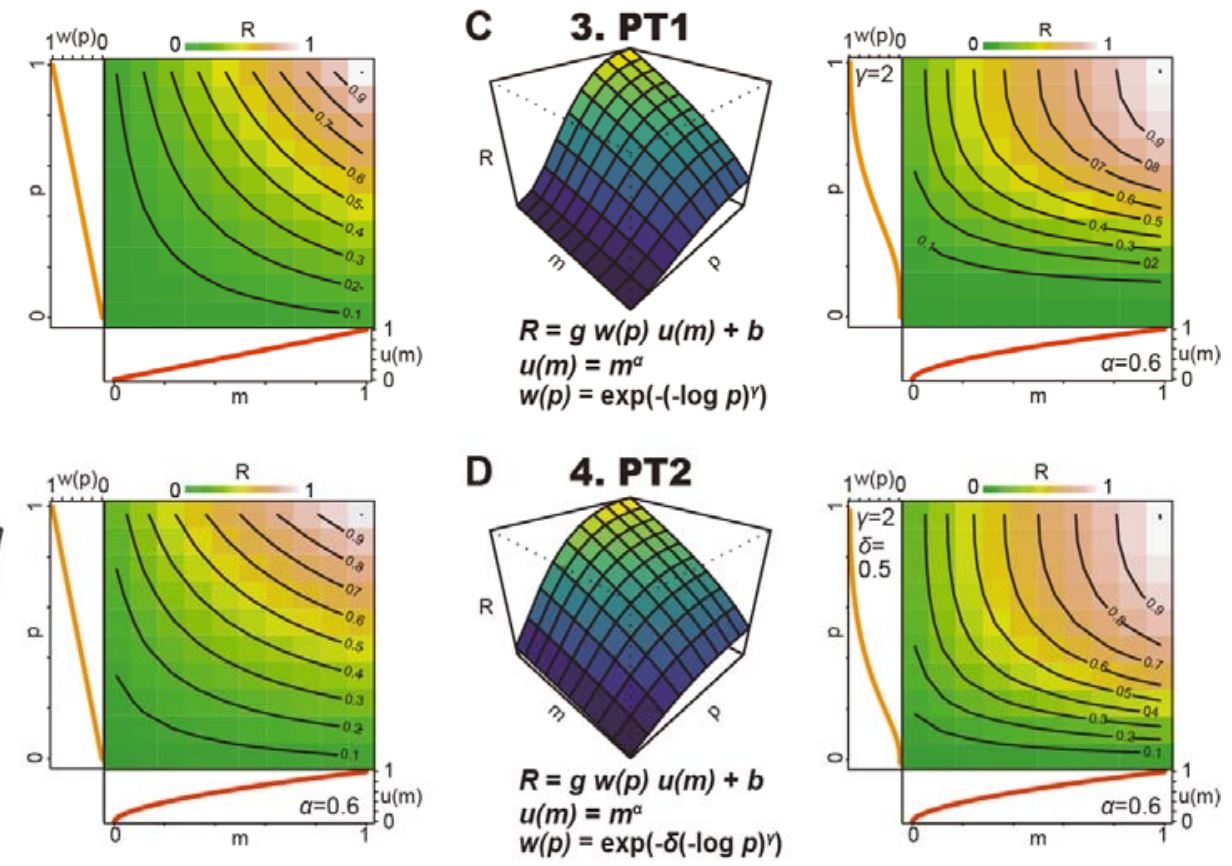

724 Figure 2. Neural models of economic decision theory

725 Schematic depiction of predicted neuronal responses $R$ defined by the four economic

726 models that represent expected value (A, EV), expected utility $(B, E U)$, and prospect

727 theory one-parameter Prelec (C, PT1) and two-parameter Prelec (D, PT2). Model

728 equations are shown in each plot. $R$ is plotted against the probability $(p)$ and magnitude

$729(m)$ of the rewards. $b, g, \alpha, \gamma$, and $\delta$ are free parameters. $g$ and $b$ are the gain and intercept

730 parameters, respectively. $\alpha$ represents the curvature of the $u(m) . \delta$ and $\gamma$ represent

731 probability weighting functions. For these schematic drawings, the following values for free

732 parameters were used: $b, g, \alpha, \gamma$, and $\delta$ were 0 spk s ${ }^{-1}, 1,0.6,2$, and 0.5 , respectively, for

733 all four figures. See Methods section for more details. 
Yamada et al.
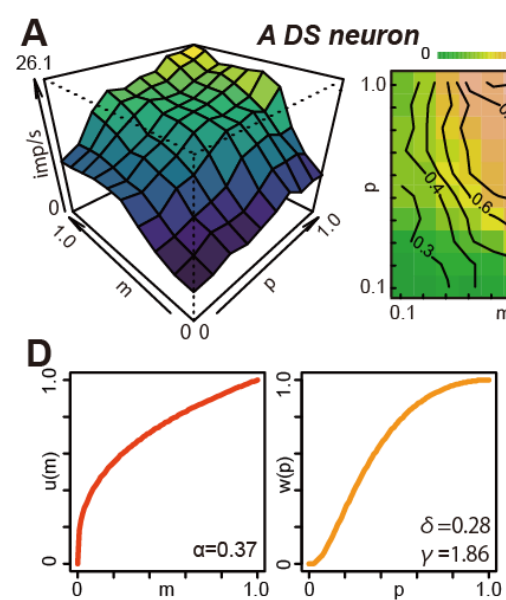
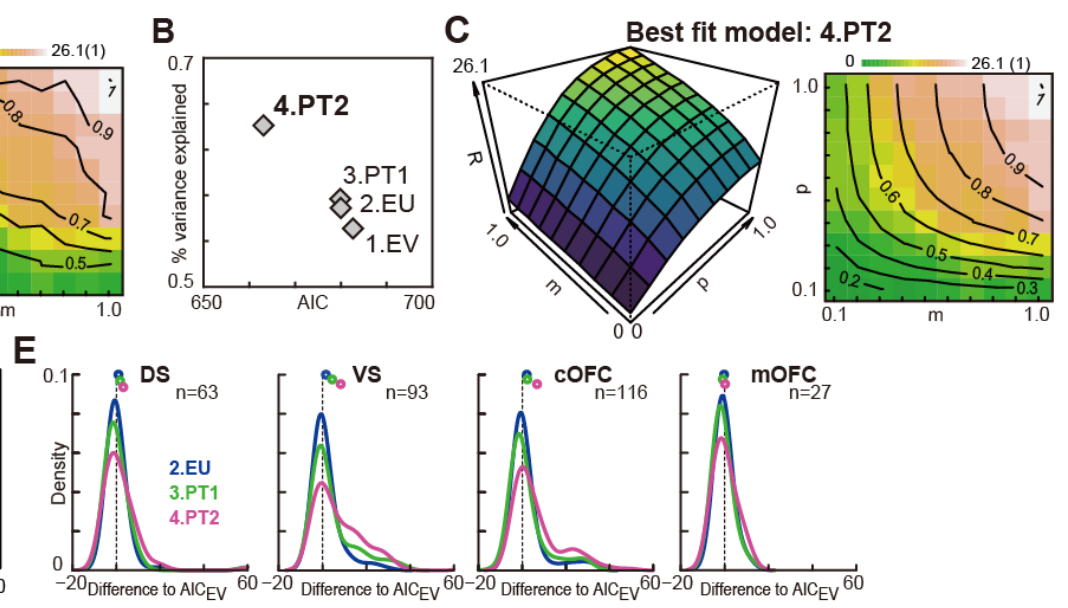

735

736

737

738

739

740

741

742

743

744

745

746

747

748

749 and magnitude of rewards. the example neuron in $(A)$.

Figure 3. Prospect theory best explained neural firing rates in the reward circuitry

(A) Plot of an example activity of the DS neuron in Figure $1 \mathrm{H}$ against probability $(p)$ and magnitude $(m)$ of rewards. To draw the 3D curvature (left) and contour lines (right), neighbouring pixels were average smoothed.

(B) The AIC values against the percent variance explained are plotted in each model for

(C) A 3D histogram (left) and contour lines (right) predicted from the best-fit PT2 model in (A). The activity of the example neuron in $(A)$ is shown in the right color map figure. Contour lines are shown for every $10 \%$ change in the fitted model.

(D) $u(m)$ and $w(p)$ estimated in the best-fit model PT2 for the neural activity in (A).

(E) Probability density of the estimated AIC difference of the three models against the EV (the simplest) model. The plots display mean values. $\mathrm{n}$ represents the number of neuronal signals that showed both positive or both negative regression coefficients for probability 
Yamada et al.
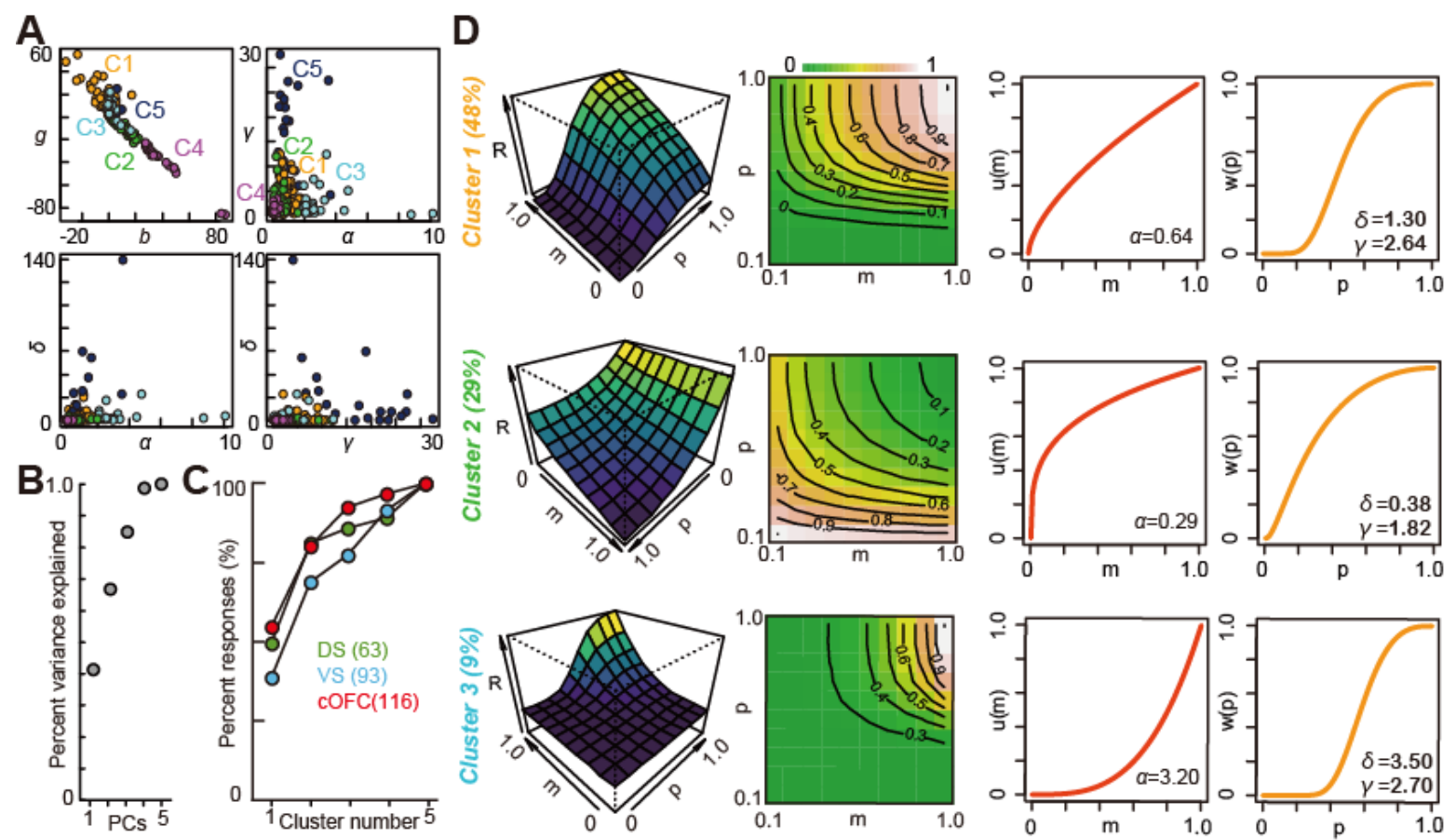

Figure 4. Neuronal clusters categorized by the fitted parameters according to the

753 prospect theory model

754 (A) Plots of all five parameters estimated in DS, VS, and cOFC neurons. $g, b, \alpha, \delta$, and $\gamma$ 755 are plotted.

756 (B) Cumulative plot of the percent variance explained by PCA is shown against the 757 principal components PC1 to PC5.

758 (C) Cumulative plot of the percentages of activity categorized into the five clusters in each 759 brain region.

760 (D) Response $R$ (model output) in the first three predominant clusters are plotted. 3D 761 curvature, contour lines with color maps, $u(m)$, and $w(p)$ are plotted using mean values of 762 each parameter in each cluster. For drawing the 3D curvature (first column) and contour 763 lines (second column), $R$ is normalized by the maximal value. 
Yamada et al.
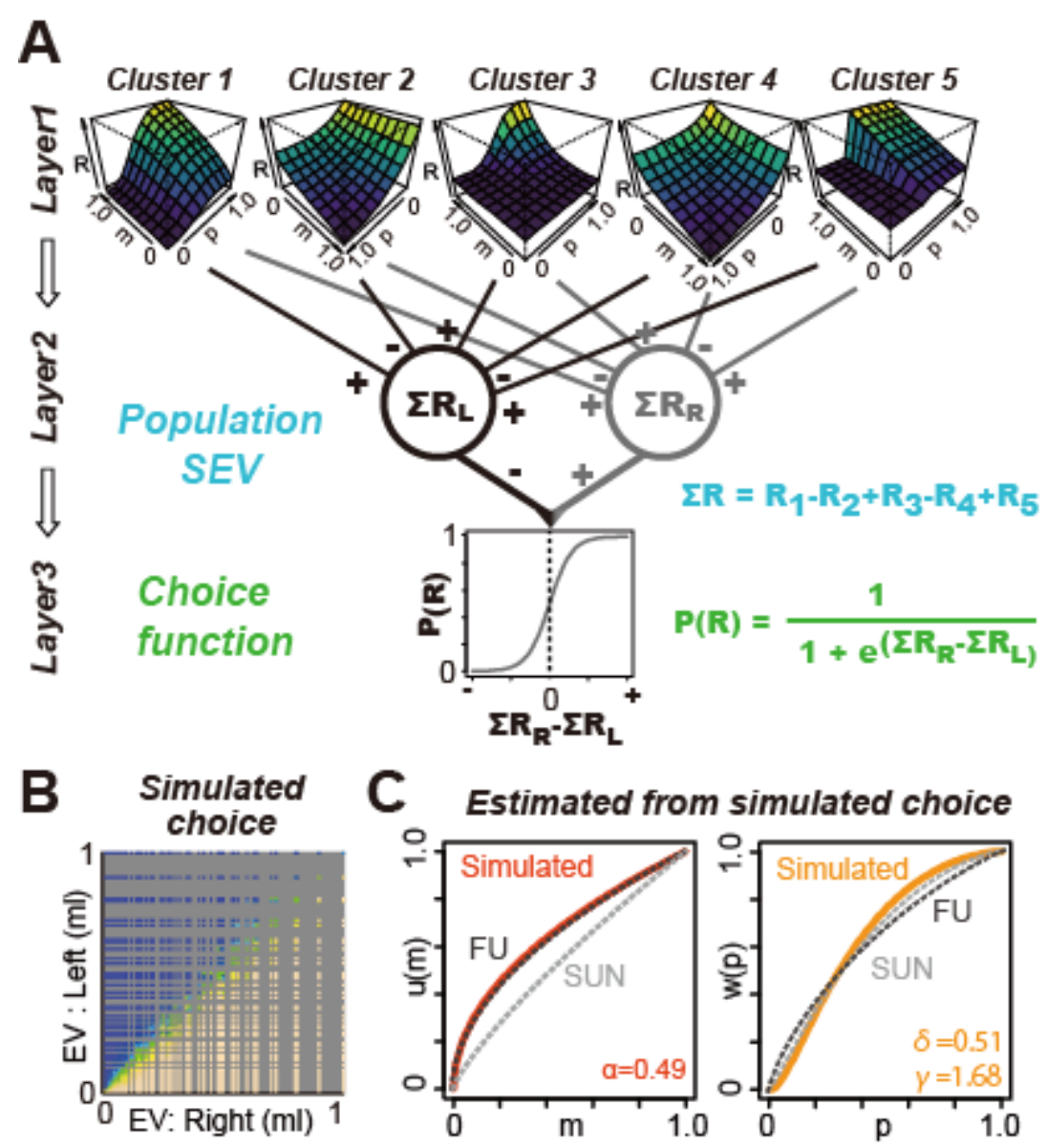

766 Figure 5. A simple network model reconstructs the subjective decision statistics in

767 monkeys

768 (A) The five neural clusters as detected by PCA in the reward circuitry. Subjective 769 expected value functions (SEVs) for left and right target options are defined as the linear

770 summation of the five clusters (see Methods). Choice is simulated as a sigmoid function of

771 the subjective value signal difference.

772 (B) The frequency with which the target on the right side was selected by a computer 773 simulation based on the network shown in $(A)$. 
bioRxiv preprint doi: https://doi.org/10.1101/2021.12.18.473272; this version posted December 20, 2021. The copyright holder for this preprint (which was not certified by peer review) is the author/funder, who has granted bioRxiv a license to display the preprint in perpetuity. It is made available under aCC-BY-NC-ND 4.0 International license.

Yamada et al.

774 (C) $u(m)$ and $w(p)$ estimated from the simulated choice in (B) are plotted. Dotted lines

775 indicate the actual functions $u(m)$ and $w(p)$ of the monkeys, as shown in Figure 1E and $1 \mathrm{~F}$, 776 respectively. 